\title{
CONTRACTIBILITY AND CONVEXITY1
}

\author{
H. W. KUHN
}

The purpose of this note is to present a purely geometrical result that appeared as a by-product of research in the theory of games. A preliminary draft had been written when it was discovered that a proof of the theorem had been published by I. Liberman [1 $]^{2}$ in 1943. However, the present proof seems to retain its interest, both for its conciseness and the fact that it exposes a connection between the geometry of convex sets and fixed-point theory that was motivated by game theory.

The result in question is:

Theorem. In order that a locally contractible compact set $X$ in $n$-dimensional Euclidean space be convex, it is necessary and sufficient that the set $X$ be contractible and that every support contact of $X$ be contractible.

By a support contact of $X$ is meant the intersection of $X$ with a supporting hyperplane, that is, the set of $x=\left(x_{1}, \cdots, x_{n}\right) \in X$ for which a given linear form $(a, x)=a_{1} x_{1}+\cdots+a_{n} x_{n}$ assumes its maximum value $\alpha$; support contacts of convex sets are obviously convex. The necessity of the conditions is clear since a convex set $X$ can always be contracted to any point $x_{0} \in X$ by the contraction $h(x, t)$ $=t x_{0}+(1-t) x$ where $x \in X$ and $0 \leqq t \leqq 1$. To prove the sufficiency of the conditions, assume that $X$ is not convex and let $C \neq X$ be the convex hull of $X$. Then there exists a point $p$ on the boundary of $C$ that does not lie in $X$ (otherwise $X$ would contain the boundary of $C$ which is a topological sphere, but not some point inside the sphere, contradicting the contractibility of $X$ ). Since $X$ is compact, $C$ is also and there exists a hyperplane supporting $C$ through $p$. Choose coordinates in the Euclidean space so that $p$ is the origin $O$ and the supporting hyperplane is given by $x_{1}+\cdots+x_{n}=0$, that is, $x_{1}+\cdots$ $+x_{n} \leqq 0$ for all $x \in C$. Relative to this choice of coordinates, consider the zero-sum two-person game played thus:

Move 1. Player I chooses a point $x \in X$.

Move 2. Player II chooses an integer $i=1, \cdots, n$.

Payoff. Player II pays Player I the amount $x_{i}$.

Presented to the Society, October 24, 1953; received by the editors March 4, 1954.

1 The preparation of this paper was sponsored (in part) by the Office of Scientific Research, USAF, and (in part) by the Logistics Project, ONR, Princeton University.

2 Numbers in brackets refer to the bibliography at the end of the paper. 
If Player II plays the integer $i$ with probability $y_{i}$, where $y_{i} \geqq 0$ and $y_{1}+\cdots+y_{n}=1$, then the expected payoff is

$$
f(x, y)=x_{1} y_{1}+\cdots+x_{n} y_{n} .
$$

Since $x_{1}+\cdots+x_{n} \leqq 0$ for all $x \in X$ and $0 \notin X$, it is clear that every $x \in X$ has at least one negative coordinate and hence

$$
\max _{x} \min _{y} f(x, y)=\max _{x} \min _{i} x_{i}<0,
$$

where the existence of the maximum is assured by the compactness of $X$. Thus the value of the game to Player I is negative. On the other hand, suppose Player II can choose probabilities $\bar{y}_{1}, \cdots, \bar{y}_{n}$ such that $\max _{x} f(x, \bar{y})=\alpha<0$. Then $(\bar{y}, x)=\alpha$ is a supporting hyperplane for $X$ and hence for $C$. However $0 \in C$ and $(\bar{y}, 0)=0>\alpha$. Hence no such $\bar{y}$ exists and

$$
\min _{y} \max _{x} f(x, y) \geqq 0 .
$$

Thus the value of the game to Player II is nonpositive, and the game does not have a value.

In a recent paper [2], Debreu has observed that fixed-point theorems of Eilenberg and Montgomery [3] or Begle [4] imply the following theorem on zero-sum two-person games:

Let $X$ and $Y$ be contractible and locally contractible compact sets in $n$-dimensional Euclidean space, and $f(x, y)$ a continuous real-valued function defined on $X \times Y$. If the sets

$$
\begin{aligned}
& U_{\bar{x}}=\left\{y \in Y \mid f(\bar{x}, y)=\min _{y} f(\bar{x}, y)\right\}, \\
& V_{\bar{y}}=\left\{x \in X \mid f(x, \bar{y})=\max _{x} f(x, \bar{y})\right\}
\end{aligned}
$$

are contractible for all $\bar{x} \in X$ and all $\bar{y} \in Y$, then

$$
\max _{x} \min _{y} f(x, y)=\min _{y} \max _{x} f(x, y) .
$$

In the language of game theory, if $X$ and $Y$ are the sets of strategies and $f(x, y)$ the payoff function for a zero-sum two-person game, then this theorem asserts that the game has a value. For the game defined above, the sets $U_{\bar{x}}$ are faces of the simplex $Y$ of probabilities $y_{1}, \cdots$, $y_{n}$ since the function $f$ is linear. On the other hand, the sets $V_{\bar{y}}$ are support contacts of $X$ and hence are contractible by assumption. Therefore the game has a value, contradicting conclusions (2) and (3) above. The assumption that $X$ is not convex falls with them and 
this completes the proof of the theorem.

Liberman's theorem is stronger than the result just proved in one important respect; it does not presuppose local contractibility. This condition entered the proof by way of the fixed-point theorems and it does not seem possible to eliminate it directly without a stronger theorem than seems to exist in the literature. Indeed, it would require the following conjectural statement:

Let $X$ be a contractible compact set in $n$-dimensional Euclidean space and $T$ an upper semi-continuous mapping which assigns to each point $x \in X$ an acyclic subset $T(x) \subset X$. Then $T$ has a fixed point, i.e., for some $x, T(x)$ contains $x$.

Although the special case of a single-valued mapping is a wellknown and long-standing problem (an example of Borsuk [5] shows that it is not enough to assume that $X$ is an acyclic compact set), the validity of Liberman's theorem seems to indicate possible progress in this direction.

(Added March 30, 1954. The discovery by S. Kinoshita of a contractible continuum in 3-dimensional Euclidean space without fixed point property (Fund. Math. vol. 40 (1953) pp. 96-98) means that Liberman's theorem cannot be proved directly by means of a fixed point theorem.)

\section{BIBLIOGRAPHY}

1. I. Liberman, On certain characteristic properties of convex bodies, Mat. Sbornik vol. 13 (55) (1943) pp. 239-262.

2. G. Debreu, $A$ social equilibrium existence theorem, Proc. Nat. Acad. Sci. U. S.A vol. 38 (1952) pp. 886-893.

3. S. Eilenberg and D. Montgomery, Fixed point theorems for multivalued transformations, Amer. J. Math. vol. 68 (1946) pp. 214-222.

4. E. G. Begle, A fixed point theorem, Ann. of Math. vol. 51 (1950) pp. 544-550.

5. K. Borsuk, Sur un continu acyclique qui se laisse transformer en lui même sans points invariant, Fund. Math. vol. 24 (1935) pp. 51-58.

Bryn Mawr College and

National Bureau of Standards, Los Angeles 